\title{
MODELAGEM MATEMÁTICA DA COPOLIMERIZAÇÃO DE ESTIRENO-DIVINILBENZENO EM SOLUÇÃO DILUÍDA
}

\author{
V. N. SANTOS, L.G. AGUIAR e R.GIUDICI \\ Universidade de São Paulo, Escola Politécnica Departamento de Engenharia Química \\ E-mail para contato: viniciusnobre@usp.br
}

\begin{abstract}
RESUMO - Neste trabalho foi feita a modelagem matemática da copolimerização de estireno com divinilbenzeno por balanços de massa para cada espécie no meio reacional, distinguindo-se os tamanhos de cadeia e adotando-se a hipótese de estado pseudo-estacionário para os radicais. No modelo foram incluídas as reações de ciclização através de um balanço de sequências. O objetivo deste trabalho foi estudar reações com baixas concentrações de monômero a fim de se evitar as reações de ligação cruzada, aumentando assim, a incidência das reações de ciclização para que se possa estimar a respectiva expressão de Arrhenius. O Modelo foi capaz de fazer previsões de conversão de monômero e concentração de duplas ligações pendentes nas cadeias poliméricas. A constante de ciclização estimada através do modelo para a menor sequência (3 unidades monoméricas) foi $250 \mathrm{~s}^{-1}$ a $60^{\circ} \mathrm{C}$ com energia de ativação de $32500 \mathrm{cal}^{\mathrm{mol}}{ }^{-1}$.
\end{abstract}

\section{INTRODUÇÃO}

Polímeros reticulados têm recebido bastante atenção desde o início dos estudos de ciência e tecnologia de polímeros devido as suas características especiais e a possibilidade de mudança em suas propriedades. Um dos exemplos mais notáveis é a copolimerização radicalar de estireno (S) e divinilbenzeno (DVB) que é um sistema classicamente estudado e amplamente utilizado para sintetizar redes poliméricas. É bem sabido que existe uma grande diferença entre a reatividade dos dois isômeros divinílicos (meta-divinilbenzeno e para-divinilbenzeno). A introdução de uma unidade de DVB em um copolímero nem sempre leva a um crosslink, podendo resultar em uma dupla ligação pendente do tipo meta ou do tipo para ( $m$ PDB ou $p$ PDB) dependendo das condições de reação. (Gonçalves, 2013).

Além disso, durante uma polimerização radicalar podem ocorrer reações paralelas que afetam a homogeneidade da microestrutura das redes poliméricas, dessas reações podemos destacar as reações de ciclização intramolecular, que ocorre entre um centro radicalar e uma dupla ligação pendente da mesma cadeia polimérica, atrasando o ponto de gel e aumentando a heterogeneidade da rede polimérica (Aguiar, 2013).

Ao longo dos anos, técnicas matemáticas com diferentes níveis de complexidade têm sido aplicadas para modelagem de reações envolvendo polímeros ramificados e algumas delas têm sido implementadas para estudar polímeros reticulados. Entretanto, na maioria dos casos os modelos são capazes de reproduzir algumas medidas experimentais, como conversão e peso molecular da fração solúvel no período pré gel, entretanto a predição do desenvolvimento das características das redes poliméricas ainda permanece como um problema de caixa-preta (Kryven, Berkenbos, 2013). 
Neste trabalho pretende-se apresentar uma abordagem mais completa para inclusão das reações de ciclização. No modelo foram utilizados balanços molares para cada espécie presente no meio reacional, distinguindo-se tanto a espécie quanto os tamanhos, adotando-se a hipótese de estado pseudo-estacionário para os radicais. As reações de ciclização foram incluídas considerando-se uma cinética diferente para cada tipo de radical presente no meio reacional, levando-se em consideração a dependência do tamanho desses radicais na cinética para este tipo de reação. As simulações foram feitas com dados experimentais presentes na literatura a fim de se obter através do ajuste de curvas a taxa de ciclização e determinar a respectiva expressão de Arrhenius para este tipo de reação.

\section{MODELAGEM MATEMÁTICA}

A abordagem utilizada neste modelo está baseada nos balanços molares utilizando as equações cinéticas para cada espécie presente no meio reacional. As reações incluídas no mecanismo desse sistema estão baseadas no trabalho realizado por Aguiar (2014) e os mecanismos estão mostrados na Tabela 1.

Tabela 1-Mecanismos considerados na modelagem

\begin{tabular}{|c|c|}
\hline Reação & Mecanismo \\
\hline Decomposição do Iniciador & $I \stackrel{k d}{\rightarrow} 2 R_{0}$ \\
\hline Iniciação & $R_{0}+M_{j} \stackrel{k i_{j}}{\rightarrow} R_{i, r}(1 \leq j \leq 3)$ \\
\hline Propagação de Monômero & $R_{i, r}+M_{j} \stackrel{k p_{i j}}{\longrightarrow} R_{j, r+1}(1 \leq i \leq 5) ;(1 \leq j \leq 3)$ \\
\hline $\begin{array}{c}\text { Iniciação de duplas ligaçães } \\
\text { pendentes de polímero morto }\end{array}$ & $R_{0}+P_{j, r} \stackrel{k i_{j}}{\rightarrow} R_{j, r}(4 \leq j \leq 5)$ \\
\hline $\begin{array}{c}\text { Propagação em dupla ligação } \\
\text { pendente de polímero morto }\end{array}$ & $R_{i, r}+P_{j, s} \stackrel{k p_{i j}}{\longrightarrow} R_{j, r+s}(1 \leq i \leq 5) ;(4 \leq j \leq 5)$ \\
\hline Ciclização & $R_{i, 4, r} \stackrel{k c m i_{r}}{\longrightarrow} R_{4, r}(1 \leq i \leq 5)$ \\
\hline Terminação por combinação & $R_{i, 5, r} \stackrel{k c p i_{r}}{\longrightarrow} R_{5, r}(1 \leq i \leq 5)$ \\
\hline
\end{tabular}

Para a modelagem foram adotadas algumas hipóteses:

(i) A terminação cíclica (terminação entre dois radicais presentes na mesma molécula) é desprezível;

(ii) Somente cadeias primárias ciclizam; 
(iii) Um radical deve ter o tamanho de pelo menos 3 unidades monoméricas para que ocorra a reação de ciclização;

(iv) As duplas ligações estão distribuídas proporcionalmente em relação ao tamanho da cada molécula e igualmente espaçadas;

A única constante de ciclização estimada neste trabalho foi a constante para a ciclização de um radical de estireno, conectado a uma $m$ PDB através de 3 unidades monoméricas, ou seja, a constante $\mathrm{kcml}_{3}$. A variação do parâmetro de ciclização em função dos diferentes tipos de radicais foram calculadas através da proporcionalidade entre as taxas de propagação intermolecular conforme mostrado na equação 1e 2 :

$$
\begin{aligned}
& k c p i_{3}=\frac{k p_{i 5}}{k p_{i 4}} k c m i_{3} \quad(1 \leq i \leq 5) \\
& k c m i_{3}=\left(k p_{i 4} / k p_{14}\right) \cdot k c m 1_{3} \quad(2 \leq i \leq 5)
\end{aligned}
$$

As constantes de ciclização para radicais com mais do que 3 unidades monoméricas foram calculadas através da equação apresentada no trabalho de Rolfes e Stepto (1990) como se segue:

$$
k c j i_{r}=\left(\frac{2}{r-1}\right)^{1,5} k c m i_{3}(1 \leq i \leq 5) \quad(j=m \text { ou } p)
$$

A “fração" de PDB's contidas em cada unidade monomérica ( $f d m$ e $f d p$ ) e a distância entre elas $\left(d p\right.$ e $d m$ ) foram calculadas, utilizando-se o $\mathrm{Q}_{1}$ (número de unidades monoméricas incorporadas a cadeia de polímero):

$$
\begin{aligned}
& \frac{d Q_{1}}{d t}=\sum_{j=1}^{3} k i_{j} \cdot R_{0} \cdot M_{j}+\sum_{i=1}^{5} \sum_{j=1}^{3} k p_{i j} \sum_{r=1}^{\infty} R_{i, r} \cdot M_{j} \\
& f d j=\frac{j P D B}{Q_{1}} \quad(j=\text { mou } p)
\end{aligned}
$$




$$
d j=\frac{r}{f d j . r}=\frac{1}{f d j} \quad(j=m \text { ou } p)
$$

As expressões para o iniciador, monômeros e radicais primários são expressões obtidas por balanços de massa convencional. Nas equações para os radicais do tipo "j" com " $r$ " unidades monoméricas $\left(R_{j, r}\right)$ e para a concentração de PDB's ( $m$ PDB e $p$ PDB), foram incluídos termos relativos as reações de ciclização. A Equação 9 mostra a expressão genérica para os radicais do tipo 1 a 3. Para os radicais do tipo 4 e 5 o equacionamento é análogo, porém devido aos termos de ciclização, o radical do tipo 4 depende do radical do tipo 5, (um radical do tipo 4 contendo uma $p$ PDB pode ciclizar transformando-se em um radical do tipo 5 e vice versa). Portanto as expressões referentes a $R_{4, r}$ e $R_{5, r}$ foram obtidas através da resolução de um sistema linear.

$$
\begin{gathered}
R_{j, r}=\frac{\sum_{i=1}^{5} k p_{i j} R_{i,(r-1)} \cdot M_{j}}{\sum_{i=1}^{3} k p_{j i} \cdot M_{i}+k p_{j 4} \cdot \sum_{s=1}^{\infty} s . f d m \cdot P_{s}+k p_{j 5} \cdot \sum_{s=1}^{\infty} s . f d p \cdot P_{s}} \quad(1 \leq j \leq 3) \\
+(k t c+k t d) . Y o+k c m j_{r} \cdot r \cdot f d m+k c p j_{r} \cdot r . f d p
\end{gathered}
$$

Os Balanços para Polímero morto $\left(P_{r}\right)$ e PDB's ( $m$ PDB e $p$ PDB) estão descritos a seguir:

$$
\begin{aligned}
& \frac{d P_{r}}{d t}=\left[\begin{array}{c}
-\sum_{i=1}^{5} k p_{i 4} \cdot \sum_{s=1}^{\infty} R_{i, s} \cdot f d m \cdot r \cdot P_{r}-\sum_{i=1}^{5} k p_{i 5} \cdot \sum_{s=1}^{\infty} R_{i, s} \cdot f d p \cdot r \cdot P_{r} \\
+\frac{1}{2} \cdot k t c \sum_{i=1}^{5} \sum_{s=1}^{r-1} R_{i,(r-s)} \cdot \sum_{j=1}^{5} R_{j, s}+k t d \cdot \sum_{i=1}^{5} R_{i, r} \cdot Y o \\
-k i_{4} \cdot f d m \cdot r \cdot P_{r} \cdot R_{0}-k i_{5} \cdot f d p \cdot r \cdot P_{r} \cdot R_{0}
\end{array}\right] \\
& \frac{d m P D B}{d t}=\left[\begin{array}{c}
k i_{2} \cdot R_{0} \cdot M_{2}+\sum_{i=1}^{5} k p_{i 2} \cdot M_{2} \cdot \sum_{r=1}^{\infty} R_{i, r}-\sum_{i=1}^{5} k p_{i 4} \cdot m P D B \cdot \sum_{r=1}^{\infty} R_{i, r} \\
-k i_{4} \cdot m P D B \cdot R_{0}-f d m \cdot \sum_{i=1}^{5} \sum_{r=1}^{\infty} k c m i_{r} \cdot r \cdot R_{i, r}
\end{array}\right] \\
& \frac{d p P D B}{d t}=\left[\begin{array}{c}
k i_{3} \cdot R_{0} \cdot M_{3}+\sum_{i=1}^{5} k p_{i 3 .} M_{3} \cdot \sum_{r=1}^{\infty} R_{i, r}-\sum_{i=1}^{5} k p_{i 5} \cdot p P D B \cdot \sum_{r=1}^{\infty} R_{i, r} \\
-k i_{5} \cdot p P D B-f d p \cdot \sum_{i=1}^{5} \sum_{r=1}^{\infty} k c p i_{r} \cdot r \cdot R_{i, r}
\end{array}\right]
\end{aligned}
$$

Os valores de todas as constantes utilizadas no modelo exceto das constantes de ciclização, as quais foram determinadas neste trabalho, foram retiradas de Aguiar et al (2014). Todas equações de espécies e grupos estão representadas em concentração molar (mol/L) e as simulações foram feitas utilizando a função ode45 do Matlab. 


\section{RESULTADOS E DISCUSSÃO}

As medidas experimentais obtidas por Hild e Okasha foram usadas para simular a copolimerização de estireno/divinilbenzeno em solução a $60^{\circ} \mathrm{C}$ através do modelo proposto neste trabalho. Neste experimento benzeno e 2,2-azoisobutyronitrila (AIBN) foram usados como solvente e iniciador, respectivamente. As concentrações iniciais de estireno, $m$-divinilbenzeno e AIBN foram 4; 0,08 e 0,08 mol/L respectivamente. Não foi adicionado $p$ DVB nesse experimento.

Neste modelo o número de equações diferenciais varia com o número máximo de unidades monoméricas consideradas $(\mathrm{N})$. Este número deve ser um valor que, a partir dele não se têm variações significativas entre os resultados das simulações para as mesmas condições iniciais, a fim de se obter um modelo realístico e que não demande muito esforço computacional.

A Figura 1 mostra que não existe diferença significativa entre os resultados das simulações para $\mathrm{N}=300, \mathrm{~N}=400$ e $\mathrm{N}=500$, portanto o parâmetro $\mathrm{N}=300$ foi ajustado para todas as simulações deste trabalho.

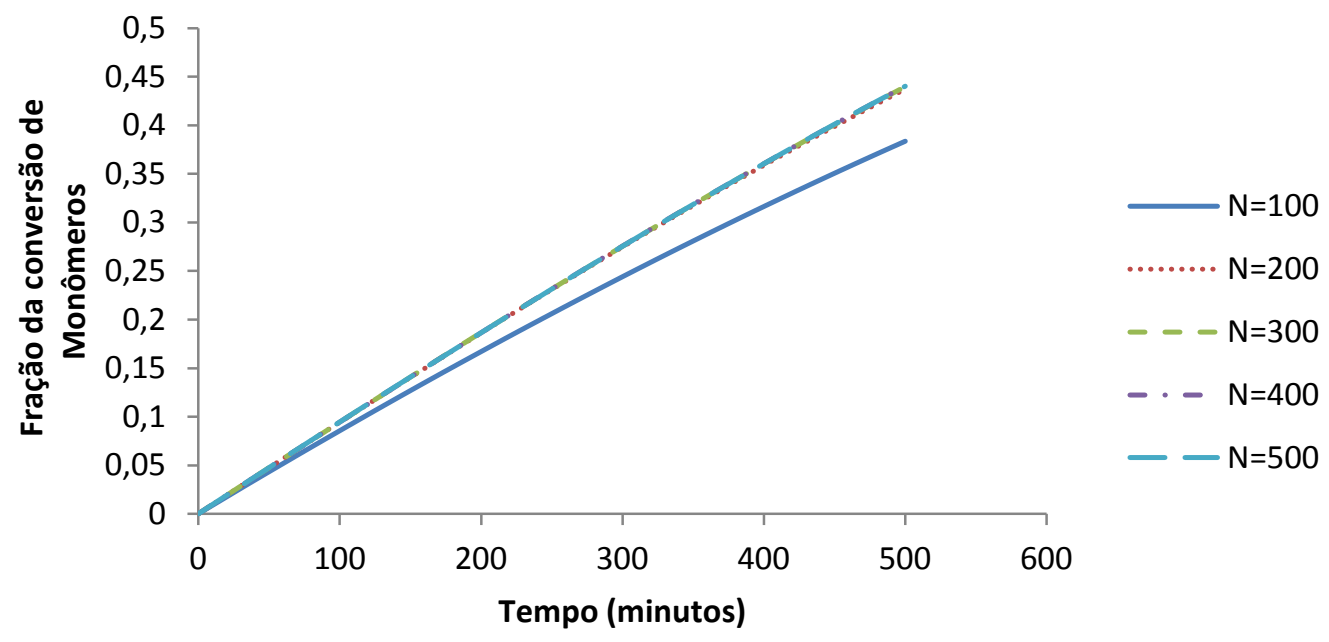

Figura 1- Conversão de Monômeros em função do tempo para diferentes números de unidades monoméricas consideradas no modelo

Na Figura 2 estão mostradas as simulações e os dados experimentais das frações relativas $[\mathrm{M}] /[\mathrm{M}]_{0}$ de monômeros estireno e $m$-divinilbenzeno e da concentração de duplas ligações pendentes. Foi estudado o efeito do parâmetro de ciclização $\mathrm{kcml}_{3}$ sobre os resultados. 

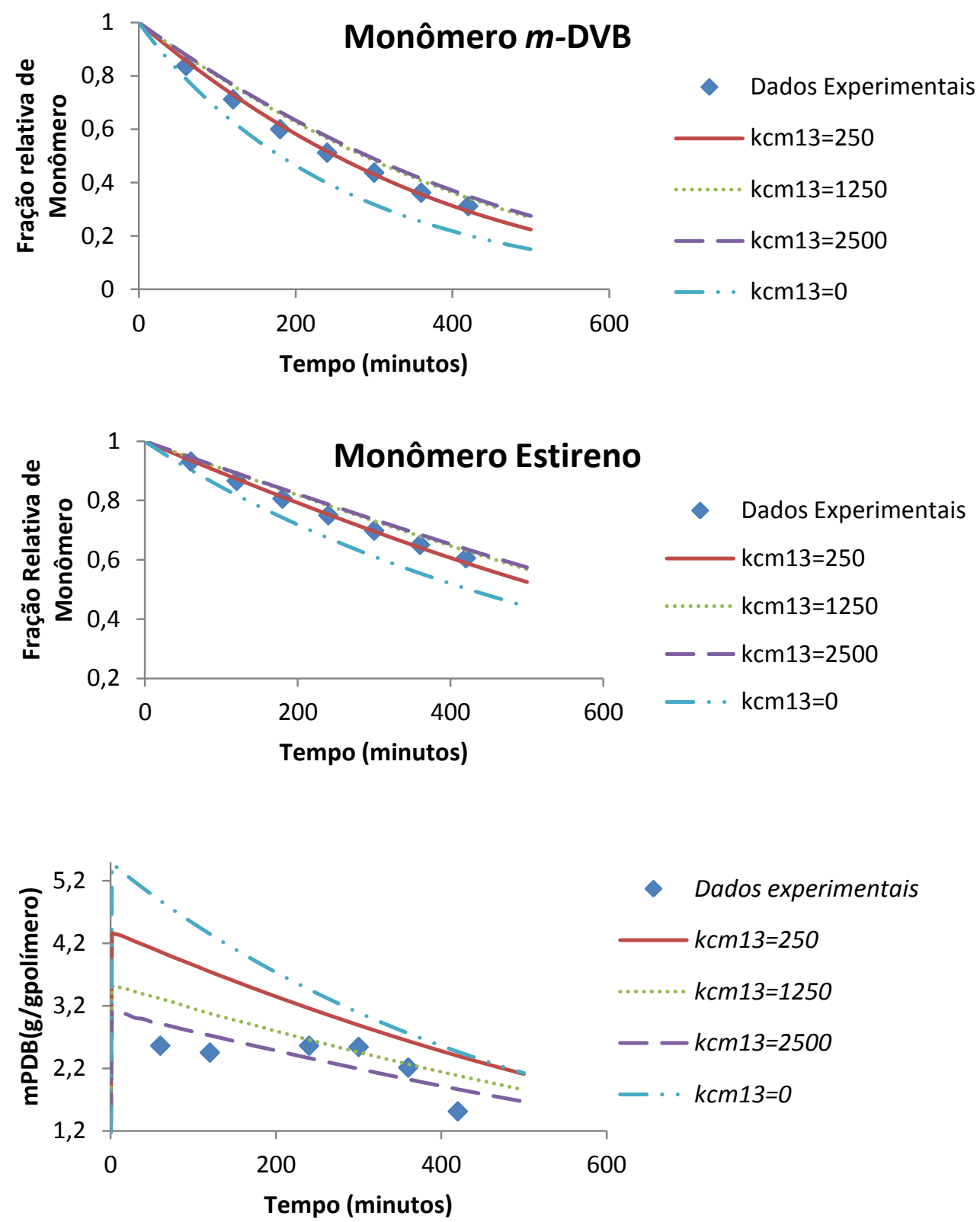

Figura 2- Curvas para conversão relativa de monômero estireno, divinilbenzeno e porcentagem em massa de PDB em função do tempo para diferentes valores de constantes de ciclização $\left(\mathrm{s}^{-1}\right) \mathrm{a}$ $60^{\circ} \mathrm{C}$.

As reações de ciclização têm uma influência indireta na conversão, pois quando ocorre este tipo de reação um radical terminal torna-se um radical no meio da cadeia polimérica diminuindo sua reatividade, consequentemente diminuindo a conversão. Isso pode ser demonstrado através dos dois primeiros gráficos da Figura 2: ao aumentar-se o valor da constante de ciclização o modelo previu menores valores de conversão. Como é sabido, as reações de ciclização consomem as PDB's diretamente. O modelo previu menores valores de concentração de PDB's ao aumentarem-se os valores das constantes de ciclização conforme esperado. 
Provavelmente melhor ajuste seria obtido se fossem retiradas algumas hipóteses do modelo como por exemplo, a negligência das ciclizações secundárias (hipótese (ii)). Apesar de se obter melhor ajuste com $\mathrm{kcml}_{3}=2500 \mathrm{~s}^{-1}$ para o perfil de $\mathrm{mPDB}$, acredita-se que o valor real é menor devido ao inesperado perfil experimental constante nos primeiros 300 minutos.

Dentre os valores de ciclização encontrados acredita-se que o valor de $\mathrm{kcm}_{3}=250 \mathrm{~s}^{-1}$ (a $60^{\circ} \mathrm{C}$ ) o qual se ajusta aos dados experimentais de conversão para o monômero estireno e para o monômero $m$-DVB seja o valor mais realista para a constante deste tipo de reação. A expressão de Arrhenius para esta reação está mostrada na Equação 11. Segundo a expressão de Arrhenius proposta por Aguiar et al. (2014), para a copolimerização de estireno/DVB mediada por nitróxido, o valor desta constante nesta temperatura é de $264 \mathrm{~s}^{-1}$.

$$
k c m 1_{3}=3,11 * 10^{7} \exp \left(-\frac{32500}{R T}\right)
$$

A Equação 11 considera que o valor da energia de ativação para a reação de ciclização (32500 J/mol) de um radical estirênico terminal com uma dupla ligação do tipo meta é o mesmo em relação a propagação do radical de estireno com o monômero estireno $k p_{11}$.

\section{CONCLUSÕES}

Foi desenvolvido um modelo cinético para a copolimerização de estireno-divinilbenzeno através de balanços de massa para cada espécie no meio reacional levando-se em conta o tamanho e o tipo de cada espécie e adotando-se a hipótese de estado pseudo-estacionário para os radicais. O modelo foi validado com dados experimentais encontrados na literatura e proveu a estimativa de $250 \mathrm{~s}^{-1}$ para a constante de ciclização para um segmento de copolímero estireno- $m$ DVB de 3 unidades monoméricas no qual é capaz de ocorrer ciclização a $60^{\circ} \mathrm{C}$. Este valor é coerente com valores encontrados na literatura, e pode ser testado em estudos futuros em condições diferentes e reajustado através da eliminação de algumas hipóteses simplificadoras que foram adotadas neste trabalho. Este método proposto representa mais um passo em direção a descrição de variáveis deste sistema e para um melhor entendimento do mecanismo de formação das redes poliméricas.

\section{NOMENCLA TURA}

\section{Concentração iniciador}

$k c j i{ }_{r}$ Constante de ciclização para o radical do tipo " $\mathrm{i}$ " contendo PDB e tamanho " $\mathrm{r}$ " ( $\mathrm{j}=\mathrm{m}$ ou p)

$k d \quad$ Constante de decomposição do iniciador

$k i_{j} \quad$ Constante de iniciação de monômero do tipo "j" para $1 \leq \mathrm{j} \leq 3, m \operatorname{PDB}$ para $\mathrm{j}=4$ e $p$ PDB se $\mathrm{j}=5$

$k p_{i j} \quad$ Constante de propagação do Radical do tipo "i”" e espécie do tipo " $\mathrm{j}$ ” $1 \leq \mathrm{j} \leq 3, m \mathrm{PDB}$ para $\mathrm{j}=4$ e $p$ PDB se $\mathrm{j}=5$

ktc Constante de terminação por Combinação 
$M_{j} \quad$ Concentração de monômero do tipo “j” (j:1= estireno, 2=m-divinilbenzeno e 3- $p$ divinilbenzeno)

$P_{j, r} \quad$ Concentração de polímero morto carregando dupla ligação pendente do tipo " j”” (j:4= $m \mathrm{PDB}, \mathrm{j}=5 \mathrm{pPDB}$ ) e tamanho " $\mathrm{r}$ " unidades monoméricas

$R_{0} \quad$ Concentração de radicais primários

Yo Concentração total de radicais

\section{REFERÊNCIAS}

AGUIAR, L.G, tese, Universidade de São Paulo, 2013. Síntese de Copolímeros EstirenoDivinilbenzeno por Polimerização Radicalar e Mediada por Nitróxido: Experimentos e Modelagem Matemática, Tese de Doutorado, Universidade de São Paulo, Brasil, 2013.

AGUIAR, L.G.; GONÇALVES M.A.D.; PINTO, V.D.; DIAS R.C.S.; COSTA, M.R.P.F.N.; GIUDICI, R., Development of Cyclic Propagation Kinectics for Modeling the Nitroxide-mediated Radical Copolymerization of Styrene-Divinylbenzene. Macromol. React. Eng, v. 8, p. 282-294, 2014.

GONÇALVES, M. A. D.; PINTO, V. D.; DIAS, R. C. S.; RUI, M.; AGUIAR, L.G.; Giudici, R. Gel Formation in Aqueous Suspension Nitroxide-Mediated Radical CoPolymerization of Styrene/Divinylbenzene. Macromol React. Eng. v. 7, p. 155-175, 2013.

HILD, G.; OKASHA, R. Kinetic investigation of the free radical crosslinking copolymerization in the pre-gel state, 1 Styre $/ m$ - and p-divinylbenzene systems. Makromol. Chem. v. 186, p. 93-110, 1984.

KRYVEN, I.; BERKENBOS, A.; MELO, P.; KIM, D.M.; Iedema,P.D. Modeling Crosslinking Polymerization in Batch and Continuous Reactors. Macromol. React. Eng., v. 7, p. 2052020, 2013.

ROLFES, H.; STEPTO, R.F.T., Network formation and properties: Rate theory description of effects of ring formation on elastic shear modulus of RA2+ RB3networks, Makromol. Chem, Macromol. Symp, v. 40, p. 61-79, 1990. 